\title{
Neutrinos from the supernova
}

\section{Physicists appear to have reacted as promptly as the better-drilled astronomers to the opportunity presented by the explosion of a supernova in the Large Magellanic Cloud on 23 February.}

THE first few weeks of Supernova 1987a have seen a flurry of IAU (International Astronomical Union) telegrams, hastily written preprints and late night telephone calls. Astronomers, already accustomed to dealing with unexpected celestial events, have mobilized with admirable speed. It is even more remarkable that physicists at underground detectors around the world have reacted with equal speed to the reported detection of neutrinos from the explosion. Now, as the supernova itself continues to shine steadily in the Large Magellanic Cloud (LMC), some of the confusion of the first few days is being dispelled.

The detection of neutrinos has created much excitement. The announcement by the Italian experiment in the Mont Blanc tunnel that neutrinos had been seen was soon followed by a conflicting report from Japan. Physicists at the Kamioka zinc mine also saw a burst of neutrinos, but four and a half hours later than the Mont Blanc events. Because a supernova can emit neutrinos only at the very instant of explosion, these reports could not both be right. Yet at the outset it was thought that no other experiment would be able to see the neutrinos (see Nature 326, 11; 1987). In particular, the high-threshold IMB (Irvine-Michigan-Brookhaven) device, situated in an Ohio salt mine, can see neutrinos only above $20 \mathrm{MeV}$, where the thermal spectrum of neutrinos from a supernova explosion should produce almost nothing.

While the discordant observations from Italy and Japan were being pondered, the IMB collaboration announced that it had in fact seen neutrinos from the supernova, and with a clear and unambiguous signal. Starting at UT 7:35:41.37 on 23 February, eight events at an energy between 20 and $40 \mathrm{MeV}$ were recorded over a period of six seconds. To within a few seconds, this is the time the neutrinos arrived in Japan, but as the Kamioka experiment does not have accurate absolute timing, the two can be regarded as simultaneous.

Although this agreement was gratifying, there was a problem. Kamioka saw just one event at $35 \mathrm{MeV}$, while IMB saw eight events of comparable energy. After a little thought, this turned out not to be a difficulty. A supernova does indeed produce fewer neutrinos at higher energy, but this is partly compensated for by the increase of neutrino interaction crosssection with the square of the energy: high-energy neutrinos are therefore more likely to be detected and for these particular neutrinos, the IMB detector is effectively seven times more sensitive than that at Kamioka.

In all this excitement, the Italian detection has rather been forgotten. The general opinion is that it saw a chance cluster of events, only a little above background noise. At both IMB and Kamioka, data have been re-examined at the time of the supposed Italian events; nothing has been found.

Meanwhile, as the euphoria of this first achievement of extragalactic neutrino astronomy subsides, physicists are trying to extract more subtle information from their data. Theoretically, at the moment of core collapse, a supernova will emit a burst of neutrinos lasting for about a second. Yet IMB and Kamioka report neutrino events over periods of six and thirteen seconds, respectively.

One explanation is that neutrinos have mass: massive neutrinos travel at less than the speed of light, so that the arrival times of neutrinos of different energy would be spread out. A simple estimate suggested that a mass of some tens of electron volts would disperse the neutrinos over a period of six seconds during their passage from the LMC. But there is a more prosaic explanation. Rather than coming directly to us from the supernova's collapsed core, the neutrinos would most probably have been scattered a few times by atoms in the stellar atmosphere on the way out, their arrival delayed by a few seconds.

In principle, the effects of neutrino mass and simple scattering can be distinguished by careful study of the energy spectrum and arrival times of the neutrinos, because the two effects depend differently on energy. People at IMB and Kamioka hope that their data will shed some light on this question, but it is unclear whether the neutrinos detected worldwide are enough to establish any useful spectral information.

The mere detection of neutrinos is proper cause for jubilation, but astronomers have been in this business longer and have more exacting standards. They continue to build up their picture of the supernova, comparing that with those of previous supernovae observed in galaxies more distant than the LMC. According to Robert Kirshner, of the HarvardSmithsonian Center for Astrophysics, SN 1987a must be categorized as Type II be- cause it has hydrogen lines in its emission spectrum, but is not altogether typical.

SN 1987a is fainter than one would expect, its light output rose more steeply than usual and has then quickly levelled out. What will happen next is not quite clear. The light curve of a typical Type II supernova rises to a maximum and then begins to fall in a matter of days. But SN 1987a does somewhat resemble two supernovae observed in the 1940 s, which, after a plateau of constant brightness, brightened again before subsiding.

Different Type II supernovae may behave differently because the early and late periods of the light curve are differently controlled. The luminosity of the initial outburst derives from the mechanical energy of the explosion, but in the later stages the energy source is the decay of radioactive nuclei, especially isotopes of cobalt synthesized in the explosion. Unusual ups and downs in the light curve may thus reveal something about the original explosion, the progress of nuclear fusion in the outburst, and the chemical composition of the progenitor star.

This progenitor has not yet been identified. The star Sanduleak-69.202, close to the explosion site, was the first and would have been the ideal candidate - except that observations by the International Ultraviolet Explorer satellite show that it is still there, as indeed are all the known stars in the vicinity. But Kirshner is not greatly concerned. He believes that the progenitor was a slightly fainter star, near the line of sight to 69.202 and obscured by it. Even so, it is irksome that the first nearby supernova for several centuries should have another star right in front of it.

SN 1987a may nevertheless be cosmologically important. It is close enough for Kirshner to hope that speckle interferometry will reveal the angular size of the expanding shell over the coming weeks and months. Knowing the brightness, expansion speed, size and distance, it will be possible accurately to reconstruct the explosion and the subsequent expansion, which in turn will help similarly to reconstruct supernovae in more distant galaxies. Because careful observations of extragalactic supernovae can provide good estimates of their distance, the potential payoff is that SN1987a will help to fix more accurately a few points on the still sparsely occupied plot of galactic redshift against distance. 